\title{
Percepção da equipe de enfermagem sobre fatores causais de quase-falhas (near miss) no atraso de medicamentos através da Análise do Tipo e Efeito de Falha (Failure Mode and Effects Analysis) - FMEA
}

\section{Perception of the nursing team about causes of near miss in the medicament delay using Failure Mode and Effects Analysis- FMEA}

\author{
Sylvia Lemos Hinrichsen ${ }^{1}$, Kaline Assis Carneiro Brayner ${ }^{2}$, Silvio Luis Lira \\ Paixão ${ }^{2}$, Tatiana de Aguiar Santos Vilella ${ }^{3}$, Marcela Coelho Lemos ${ }^{4}$, Ewerton \\ Douglas da Silva ${ }^{5}$
}

Trabalho realizado no Núcleo de Ensino, Pesquisa e Assistência em Infectologia da Universidade Federal de Pernambuco (UFPE). Disciplina de Biossegurança e Controle de Infecções Risco Sanitário Hospitalar- Recife (PE), Brasil.

\author{
1.Médica Infectologista; Coordenadora do Núcleo de Ensino, Pesquisa e Assistência em \\ Infectologia da UFPE; Professora Titular do Departamento de Medicina Tropical da UFPE- \\ Recife (PE), Brasil. \\ 2. Enfermeiro; Assistente de pesquisas do Núcleo de Ensino, Pesquisa e Assistência em \\ Infectologia da UFPE- Recife (PE), Brasil. \\ 3. Farmacêutica; Biomédica; Assistente de pesquisas do Núcleo de Ensino, Pesquisa e \\ Assistência em Infectologia da UFPE- Recife (PE), Brasil.
}

4. Aluna do curso de graduação de Biomedicina da UFPE; Assistente de pesquisas do Núcleo de Ensino, Pesquisa e Assistência em Infectologia da UFPE- Recife (PE), Brasil.

5.Aluno do curso de Educação Física da Uninassau; Assistente de pesquisas do Núcleo de Ensino, Pesquisa e Assistência em Infectologia da UFPE- Recife (PE), Brasil.

Endereço para correspondência: Sylvia Lemos Hinrichsen - Rua Jornalista Guerra de Holanda, 158/2601 -. Casa Forte - CEP: 52061-010 - Recife (PE), Brasil - E-mail:

sylviahinrichsen@hotmail.com

Fonte de financiamento: não se aplica

Conflito de interesse: não existe conflito de interesse

Recebido em: 02/10/2016

Aprovado e publicado: 21/11/2016 


\title{
RESUMO
}

A enfermagem é responsável pelas últimas etapas do processo de administração de medicamentos, podendo identificar os eventos e evitá-los, com objetivos de administrar medicamentos de forma correta. Para isto, é preciso identificar os tipos de eventos que possam levar a quase-falhas (near miss) assim como, os fatores de risco na ocorrência destes, que podem finalizar com erros. O objetivo deste estudo foi o de identificar os fatores causais de eventos (quase-falhas/ near miss) no ciclo da administração medicamentos. Realizou-se uma pesquisa quantitativa, descritiva, observacional, exploratória, pelo serviço de enfermagem e núcleo de segurança do paciente de um hospital terciário, de alta complexidade, localizado em Recife, que monitorou não conformidades no uso de medicamentos a partir da sua prescrição para o paciente. Foram observadas 5.037 não conformidades no ciclo do medicamento, a partir da prescrição eletrônica com permissão de inclusões manuais, relacionadas ao atraso de medicamento, segundo a percepção da enfermagem. Em relação aos dados obtidos através do FMEA (Análise do Tipo e Efeito de Falha) das não conformidades identificadas, foi considerado inaceitável a prática de prescrição manual na prescrição eletrônica. É fundamental que existam políticas e estratégias de melhorias que aumentem a segurança do ciclo dos medicamentos, especialmente, no atraso destes, que podem gerar quasefalhas (near miss) nas instituições que usam prontuário manual e principalmente eletrônico. As estratégias somente estarão coerentes quando houver uma cultura de segurança na medicação e na melhoria da qualidade assistencial prestada à sua clientela e pacientes.

Palavras-chave: FMEA; erros de medicamentos; quase-falha (near miss); prontuário eletrônico.

\begin{abstract}
Nursing is responsible for the final stages of the drug delivery process and can identify events avoiding them with goals to administer medication correctly. To this we must identify the types of events that could lead to near-failures (near miss) as well as the risk factors in the occurrence of these, which may end with errors. The aim of this study was to identify the causal factors of events (near miss) in the cycle of medications .It is a quantitative, descriptive, observational, exploratory study, conducted by the nursing service and patient safety department of a tertiary hospital, with high complexity, located in Recife, Brazil, who monitored non-conformities in the use of medication from patient electronic prescription. There were observed 5,037 non-conformities in the medicine cycle, from electronic prescribing with manual permissions in medical records related to the delay of medicine, according to the perception of nursing. In relation to data obtained by the FMEA (Failure Mode and Effects Analysis) of non-compliances identified, related to the delay of medication, was considered unacceptable manual prescribing practice in electronic prescription. It will be necessary the implementation of policies and strategies for medicine
\end{abstract}


prescription improvement to increase the safety of the medicine cycle, especially in the delay of these, which can cause near-failures (near miss) in the institutions that use manual records and mostly electronic. The strategies will be consistent only when there medicine prescribing safety culture and quality of care provided to their customers and patients.

Key words: FMEA; medication errors; near-failures(near-miss); electronic medical records 


\section{INTRODUÇÃO}

A administração medicamentosa numa instituição de saúde é um processo bastante complexo e de caráter multidisciplinar, com objetivos comuns de prestar assistência de qualidade, com segurança e eficácia ao cliente/paciente ${ }^{1-6}$.

No entendimento do conceito de um evento adverso, é importante saber que: 1-reação adversa é definida como qualquer injúria devido à medicação em doses normalmente utilizadas no homem para profilaxia, diagnóstico ou tratamento de uma enfermidade;2- erro de medicação é definido como uma falha no processo de medicação, podendo ser na prescrição, dispensação, preparo, administração e monitoramento; 3-erros potenciais (quase-falhas/ near miss) são eventos ocorridos, porém não causaram algum dano ao paciente, pois o erro foi interceptado antes de prejudicar o cliente/paciente ou a dose foi administrada e não causou nenhuma injúria ${ }^{2,7-9}$.

A National Coordinating Council for Medication Error Reporting and Prevention, define erro de medicação como qualquer evento evitável que, de fato ou potencialmente, conduz ao uso inadequado de medicamento ${ }^{2,8,9}$. E, eventos adversos são considerados como a ocorrência imprevista, indesejável ou potencialmente perigosa na instituição de saúde e quase-falha (near miss) como qualquer variação de um processo que não afeta um resultado, mas cuja recorrência acarreta grande chance de uma consequência adversa grave, enquadrando-se na definição de um evento adverso ${ }^{2,4,9,10}$.

A American Society of Hospital Pharmacists recomenda que para evitar erros na medicação é importante que haja: 1 - prescrição eletrônica; 2 - utilização de código de barras para medicamentos e identificação do paciente; 3 dispensação por dose unitária; 4 - preparação de medicação intravenosa pela farmácia; 5 - notificação de eventos adversos; 6 - interação multidisciplinar (farmácia, médicos e enfermeiros) e 7 - revisão da prescrição por farmacêuticos ${ }^{7,9,11,12}$.

Sabe-se que a enfermagem é responsável pelas últimas etapas, o preparo e a administração de medicamentos, mas que ela pode detectar alguma falha e parar todo processo, embora isso muitas vezes não aconteça, sendo normalmente colocada a culpa do evento à enfermagem, o que aumenta bastante a sua responsabilidade ${ }^{3,10}$.

Os erros e ou quase-falhas (near miss) nem sempre são por falha humana, mas podem ser decorrentes da falta de processos sistematizados pela instituição de saúde/hospital. E, de um modo geral, nem sempre são relatados devido ao medo que traz aos profissionais envolvidos de equipes multidisciplinares, consequentes às medidas administrativas, que vão desde punições verbais, escritas, demissões, processos civis, legais e éticos ${ }^{1,4,5,9,10,13}$. 
Na maioria das vezes, o erro ou uma quase-falha (near miss) de medicação só é identificado quando o paciente apresenta alguma manifestação clínica após a administração, ou algum dano foi percebido ao cliente ou quando houve atrasos na prescrição. Desta maneira, nem sempre serão visualizadas não conformidades técnicas, assim como outros fatores ${ }^{1,5,9,13-16}$.

Na ocorrência de uma não conformidade, como uma quase-falha (near miss), que antecede a um erro, o enfermeiro deve iniciar não só um monitoramento, mas, também uma investigação criteriosa que evidencie todas as etapas do ciclo da prescrição do medicamento, que incluam: horário, pessoal envolvido, turno, tipo de erro, estágio do processo que ocorreu a não conformidade e possíveis falhas no sistema a fim de corrigir e preveni-los, e não apenas atribuir uma culpabilidade pessoal, que na realidade poderá ser sistêmica ${ }^{9,15,16}$.

Neste sentido, o presente estudo foi programado para realizar uma análise do processo de administração medicamentosa ao paciente, relacionado a atrasos de medicamentos, na percepção da enfermagem, a fim de oferecer evidências para a prevenção de eventos e ou não conformidades, garantindo a implementação de processos sistematizados, focados na segurança do paciente.

O objetivo deste estudo foi identificar os tipos de quase-falhas (near miss) ${ }^{9}$ e os fatores de risco que ocorrem durante o processo de administração de medicamentos, na percepção da enfermagem, especificamente, nos atrasos de prescrição, por serem estes uma não conformidade mais prevalente na instituição para criar uma matriz de tolerância, segundo Análise do Tipo e Efeito de Falha (Failure Mode and Effects Analysis).

\section{METODOLOGIA}

Realizou-se uma abordagem quantitativa, descritiva, observacional e exploratória, no período de abril a junho de 2016, nas unidades assistenciais de um hospital privado, terciário, de alta complexidade, localizado em Recife, com 158 leitos, sendo 38 de terapia intensiva (adulto, coronária, neonatal e pediátrica), com uma taxa média de ocupação de $72 \%$, e, que desde 2012 utiliza o modelo de prescrição eletrônica com permissão de registros manuais, em todas as suas unidades.

O estudo foi desenvolvido no período de abril a junho de 2016 através dos resultados obtidos pela equipe de enfermagem e riscos, que implementam ações multidisciplinares para: 1 - identificar as ferramentas da implantação da cultura da qualidade e segurança do paciente; 2 - definir os componentes de uma comissão multidisciplinar de gerenciamento de riscos/ qualidade; 3 identificar os resultados/ indicadores de gestão a partir do diagrama (escopo) da gestão de risco (clínico e não clínico) e padrões de qualidade assistenciais a serem monitorados; e, 4 - realiza estudos de coortes, retrospectivos e 
prospectivos do tipo descritivo e observacional dos riscos e ou infecções identificados e ou potencialmente existentes na instituição ${ }^{9,15,16}$.

As informações obtidas estavam contidas nos registros de investigação, a partir de notificações de não conformidades observadas no processo assistencial, monitorado pelas equipes de enfermagem e do núcleo de segurança do paciente da instituição, com objetivo de identificar o ciclo do medicamento a partir de dados relacionados a atrasos dos medicamentos, através de buscas ativas e passivas de não conformidades de prescrição do paciente, e considerado pela instituição como quase-falha (near miss).

Utilizaram-se como definições: 1 - evento adverso: ocorrência imprevista, indesejável ou potencialmente perigosa na instituição de saúde; 2 - evento sentinela: ocorrência inesperada que implique em morte ou perda grave e permanente de função; 3 - erro de medicação: qualquer evento evitável que possa levar a um uso inadequado de um medicamento ou que ponha em risco a segurança do paciente, com potencialidade de ser um evento sentinela; 4 quase-falha (near miss): qualquer variação de um processo que não afeta um resultado, mas cuja recorrência acarreta grande chance de uma consequência adversa grave, enquadrando-se na definição de um evento adverso ${ }^{9,15,16}$.

Os dados estudados foram os relacionados a um período de três meses (maio a junho de 2016). As não conformidades de atrasos de medicamentos foram identificadas e selecionadas qualitativamente e quantitativamente, segundo variáveis pré-determinadas associadas ao ciclo da prescrição eletrônica do paciente, que foram observadas como as não conformidades mais usuais na instituição: 1 - prescrições manuais (acrescidas pelo prescritor ao impresso eletrônico); 2 - acréscimos manuais (inseridos pelo prescritor no impresso eletrônico); 3 - prescrições vencidas (fora do prazo de 24 horas, quando deveriam ter sido substituídas pelo prescritor); 4 - prescrição não padrão (em formulário não institucional, pessoal do prescritor); 5 - prescrição não checada (equipe de enfermagem não realizou aprazamentos).

Realizou-se, após a consolidação dos dados coletados de causas de atrasos de medicamentos na percepção da enfermagem, uma análise de modo e efeito de falha (Failure Mode and Effect Analysis)- FMEA, das não conformidades identificadas durante o período do estudo, com objetivos de criar uma matriz de tolerância, para gerar padrões de não aceitabilidade que fundamentassem políticas institucionais de melhorias para evitar o risco de atrasos de medicamentos, considerados como quase-falhas (near miss), mas, que pela continuidade de ocorrências, tornam-se erros ${ }^{16,17}$.

A metodologia de Análise do Tipo e Efeito de Falha (Failure Mode and Effects Analysis), conhecida como FMEA, é uma ferramenta que busca, em princípio, evitar, por meio da análise das falhas potenciais e propostas de ações de melhoria, que ocorram falhas no projeto do produto ou do processo. Detecta as falhas antes, diminuindo as chances de o produto ou processo falhar ${ }^{17}$. São tipos de FMEA: 1 - FMEA de produto, análise cujo objetivo é evitar falhas no 
produto ou no processo decorrente do projeto, comumente denominada também de FMEA de projeto; 2 - FMEA de processo, análise cujo objetivo é evitar falhas do processo, tendo como base as não conformidades do produto com as especificações do projeto; e 3 - FMEA de procedimentos administrativos, na qual se analisam as falhas potenciais de cada etapa do processo com o mesmo objetivo que as análises anteriores, ou seja, diminuir os riscos de falha ${ }^{17}$. Pode-se aplicar a análise FMEA nas seguintes situações: 1 - para diminuir a probabilidade da ocorrência de falhas em projetos de novos produtos ou processos; 2 - para diminuir a probabilidade de falhas potenciais (ou seja, que ainda não tenham ocorrido) em produtos/ processos já em operação; 3 - para aumentar a confiabilidade de produtos ou processos já em operação por meio da análise das falhas que já ocorreram; e para diminuir os riscos de erros e aumentar a qualidade em procedimentos administrativos ${ }^{16,17}$

No presente estudo, utilizou-se o FMEA de processo, análise cujo o objetivo é o de evitar falhas do processo, tendo como base as não conformidades do produto com as especificações do projeto, assim com o objetivo de análise de procedimentos administrativos, na qual se analisam as falhas potenciais de cada etapa do processo com o mesmo objetivo que as análises anteriores, com fins de diminuir os riscos de falha ${ }^{16,17}$.

Na montagem do FMEA, consideraram-se as principais etapas do processo de produção, envolvendo manipulação, análise e compreensão. Como função, determinou-se a utilidade de cada etapa diante das etapas posteriores. Como modo de falha, consideraram-se as falhas apontadas nas planilhas de não conformidades. Como efeito da falha, evidenciaram-se as consequências de cada modo de falha tanto para os clientes internos como externos. Para a determinação de possíveis eventos causadores dos modos de falha, utilizou-se de experiência prática e literatura técnica especializada ${ }^{16,17}$.

A seguir, classificou-se cada modo de falha nos critérios de ocorrência e gravidade, atribuindo notas. Quanto maior a gravidade e ocorrência, maiores as notas , considerando: Trivial/ Mínima Criticidade 1 - Não requer ação específica; Aceitável/ Pequena Criticidade 2, 3 - Não é necessário melhorar a ação, no entanto, devem ser consideradas avaliações periódicas a fim de analisar sua evolução, pois afeta, mas não desqualifica, por não ser tão significativo; Moderado Criticidade 4, 5, 6 - Devem ser implementadas políticas de redução do risco a médio e curto prazo, pois afeta, não desqualifica, mas leva a uma investigação; Importante/Alta Criticidade 7, 8, 9 - O risco precisa ser reduzido rapidamente. Devem ser implementadas políticas de redução do risco a curto prazo e avaliações periódicas para garantia da redução do risco; Inaceitável Criticidade 10,12-16 - Ação não deve continuar até que o risco seja imediatamente reduzido, sob a pena de serem cancelados atos/procedimentos clínicos e até encerrados os serviços clínicos ${ }^{16,17}$.

$\mathrm{Na}$ estratégia de análise do tipo e efeito de falha de análise dos riscos (Índice de ocorrência da causa) utilizou-se: 1 - Índice; 2 - Risco/ Probabilidade de ocorrência; e, 3 - Ocorrência que variaram entre si: 1 Muito improvável/ remota 
Excepcional; 2 Muito pequena/ improvável Muitas poucas vezes; 3 Pequena/ Provável Poucas vezes; 4, 5, 6 Moderada/ Muito provável Ocasionalmente, algumas vezes; 7, 8 Alta Frequente; 9, 10, 12-16 Muito Alta Inevitável, certamente ocorrerá falha ${ }^{16,17}$.

Cada índice foi avaliado separadamente, atribuindo notas de acordo com a experiência dos envolvidos no processo, considerando o impacto que a falha acarretaria para a próxima etapa e contando com a ajuda de outros técnicos que atuam na mesma área. Após a avaliação dos índices, calculou-se o índice de risco pelo registro do produto dos três índices anteriores: gravidade, ocorrência e detecção. Uma vez calculado, obteve-se vários índices de riscos que foram desenhados numa matriz de tolerância. Os índices que apresentaram maiores valores foram considerados como problemas a serem priorizados e sobre os quais deveria ser estabelecido um plano de ação pelas equipes e governança ${ }^{16,17}$.

O projeto teve a aprovação da Comissão de Ética em Pesquisa Envolvendo Seres Humanos- Plataforma Brasil (CAAE: 45221015.1.0000.5208).

\section{RESULTADOS}

Durante o período do estudo observaram-se 5.037 não conformidades no ciclo do medicamento, a partir da prescrição eletrônica com permissão de registros manuais no prontuário, relacionado ao atraso de medicamento, segundo a percepção da enfermagem (Quadro 1).

Em relação aos dados obtidos através do FMEA das não conformidades identificadas, relacionadas ao atraso de medicamento, foi considerado inaceitável a prática de prescrição manual na prescrição eletrônica (Quadros 1, 2 e Figura 1). 
Quadro 1. Não conformidades identificadas no ciclo do medicamento, segundo quase-falha (near miss) relacionadas a atrasos de prescrição. Recife, 2016.

\begin{tabular}{|l|r|}
\hline Não conformidades identificadas & Frequência (n) \\
\hline Mês de Abril & 811 \\
\hline Prescrições manuais & 636 \\
\hline Acréscimos manuais & 273 \\
\hline Prescrições vencidas & 66 \\
\hline Prescrições não padrão & 28 \\
\hline Prescrições não checadas & $\mathbf{1 8 1 4}$ \\
\hline Total & 826 \\
\hline Mês de Maio & 549 \\
\hline Prescrição manual & 203 \\
\hline Acréscimo manual & 119 \\
\hline Prescrição vencida* & 17 \\
\hline Prescrição não padrão & $\mathbf{1 7 1 4}$ \\
\hline Prescrição não checada & 751 \\
\hline Total & 478 \\
\hline Mês de Junho & 165 \\
\hline Prescrição manual & 111 \\
\hline Acréscimo manual & 04 \\
\hline Prescrição vencida & $\mathbf{1 5 0 9}$ \\
\hline Prescrição não padrão & \\
\hline Prescrição não checada & \\
\hline Total & \\
\hline
\end{tabular}

(*)1-Prescrições manuais (acrescidas pelo prescritor ao impresso eletrônico); 2-acréscimos manuais (inseridos pelo prescritor no impresso eletrônico); 3-prescrições vencidas (fora do prazo de 24 horas, quando deveriam ter sido substituídas pelo prescritor por novas); 4prescrição não padrão(em formulário não institucional, pessoal do prescritor); 5- prescrição não checada (equipe de enfermagem não realizou aprazamentos). 
Quadro 2. FMEA. Análise do Tipo e Efeito de Falha. Atraso de medicamentos. Recife, 2016

\begin{tabular}{|c|c|c|c|c|c|c|}
\hline \multicolumn{7}{|l|}{ FMEA } \\
\hline$\frac{2}{\mathbb{2 \pi}}$ & $\begin{array}{l}\text { Eventos } \\
\text { Adversos } \\
\text { Definidos para } \\
\text { Cada Gestão }\end{array}$ & Danos & $\begin{array}{l}\text { Gravidade do } \\
\text { Dano } \\
\text { Ligeira (1) } \\
\text { Grave (2) } \\
\text { Muito Grave (3) } \\
\text { Morte(4) }\end{array}$ & $\begin{array}{l}\text { Probabilidade } \\
\text { Muito } \\
\text { improvável (1) } \\
\text { Improvável (2) } \\
\text { Provável (3) } \\
\text { Muito Provável } \\
\text { (4) }\end{array}$ & $\begin{array}{l}\text { Estimar } \\
\text { Risco } \\
\text { (R=GxP) }\end{array}$ & Risco \\
\hline \multirow{7}{*}{ 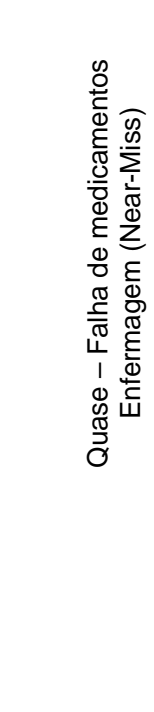 } & \multirow{4}{*}{$\begin{array}{l}\text { Prescrição } \\
\text { Manual }\end{array}$} & $\begin{array}{l}\text { Perda de } \\
\text { rastreabilidade } \\
\text { pela farmácia }\end{array}$ & 3 & 2 & 6 & Moderado \\
\hline & & $\begin{array}{l}\text { Atraso em } \\
\text { administração de } \\
\text { medicamentos }\end{array}$ & 3 & 3 & 9 & Importante \\
\hline & & |llegibilidade & 3 & 4 & 12 & Inaceitável \\
\hline & & $\begin{array}{l}\text { Administração } \\
\text { medicação errada }\end{array}$ & 4 & 2 & 8 & Importante \\
\hline & \multirow[t]{3}{*}{$\begin{array}{l}\text { Acréscimo } \\
\text { Manual }\end{array}$} & $\begin{array}{l}\text { Perda } \\
\text { rastreabilidade }\end{array}$ & 3 & 3 & 9 & Importante \\
\hline & & $\begin{array}{l}\text { Atraso em } \\
\text { administração de } \\
\text { medicamentos }\end{array}$ & 3 & 3 & 9 & Importante \\
\hline & & |llegibilidade & 2 & 3 & 6 & Moderado \\
\hline $\begin{array}{l}\frac{2}{\pi} \\
\frac{10}{4} \\
0\end{array}$ & $\begin{array}{l}\text { Eventos } \\
\text { Adversos } \\
\text { Definidos para } \\
\text { Cada Gestão }\end{array}$ & Danos & $\begin{array}{l}\text { Gravidade do } \\
\text { Dano } \\
\text { Ligeira (1) } \\
\text { Grave (2) } \\
\text { Muito Grave (3) } \\
\text { Morte(4) }\end{array}$ & $\begin{array}{l}\text { Probabilidade } \\
\text { Muito } \\
\text { improvável (1) } \\
\text { Improvável (2) } \\
\text { Provável (3) } \\
\text { Muito Provável } \\
\text { (4) }\end{array}$ & $\begin{array}{l}\text { Estimar } \\
\text { Risco } \\
\text { (R=GxP) }\end{array}$ & Risco \\
\hline \multirow{5}{*}{ 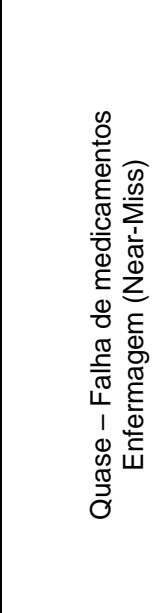 } & \multirow{2}{*}{$\begin{array}{l}\text { Prescrição } \\
\text { Vencida }\end{array}$} & $\begin{array}{l}\text { Administração } \\
\text { medicação } \\
\text { desnecessária }\end{array}$ & 2 & 2 & 4 & Moderado \\
\hline & & $\begin{array}{l}\text { Administração de } \\
\text { medicação por } \\
\text { superdosagem } \\
\text { ou não } \\
\text { administração da } \\
\text { medicação }\end{array}$ & 3 & 3 & 9 & Importante \\
\hline & $\begin{array}{l}\text { Prescrição Não } \\
\text { Padrão }\end{array}$ & $\begin{array}{l}\text { Perda de } \\
\text { rastreabilidade }\end{array}$ & 3 & 2 & 6 & Moderado \\
\hline & $\begin{array}{l}\text { Prescrição Não } \\
\text { Checada }\end{array}$ & $\begin{array}{l}\text { Administração } \\
\text { com } \\
\text { superdosagem }\end{array}$ & 3 & 3 & 9 & Importante \\
\hline & & & & & & \\
\hline
\end{tabular}




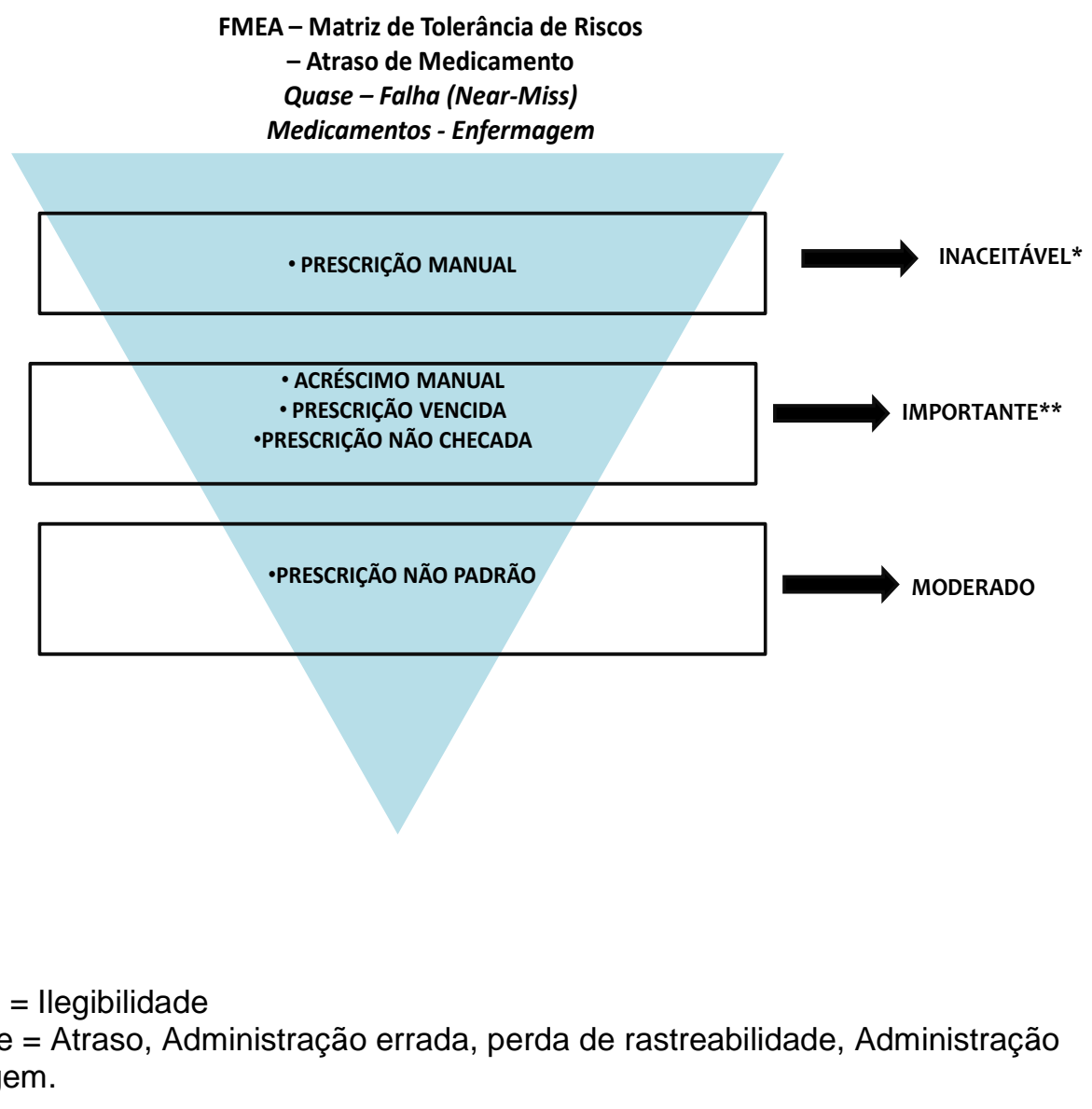

Figura 1. FMEA. Análise do Tipo e Efeito de Falha. Matriz de Tolerância de Riscos. Atrasos de Medicamentos. Recife, 2016.

\section{DISCUSSÃO}

Os erros de medicação ainda são um problema mundial de saúde pública, sendo os mais graves os relacionados aos de prescrição ${ }^{1-8,10-13,18}$.

Ainda há a necessidade da padronização no processo de prescrição e a eliminação das feitas à mão, e várias são as alternativas visando diminuir erros e ou quase-falhas (near miss) de prescrições ${ }^{18}$.

$\mathrm{Na}$ tentativa de minimizar erros de prescrição, as recomendações com maior evidência científica para a prevenção de erros e ou quase-falhas (near miss) de medicação em hospitais são: 1 - adoção da prescrição eletrônica com o devido suporte clínico; 2 - inclusão de farmacêuticos nas visitas clínicas; 3 viabilização de contato com farmacêuticos durante 24 horas para solucionar RAS - Vol. 17, № 66 - Jan-Mar 2017 
dúvidas em relação a medicamentos; e 4 - presença de procedimentos especiais e protocolos escritos para o uso de medicamentos potencialmente perigosos $^{19}$.

A implantação da prescrição eletrônica pode ter forte impacto nos erros de prescrição, devendo ser buscada na instituição, tem um alto custo de implantação, além de trazer dificuldades na sua aceitabilidade por parte dos prescritores, por hábitos, culturas e ou agendas, que dificultam a sua utilização como esperado ${ }^{19,20}$.

Entre os vários aspectos existentes na literatura para prevenir ou reduzir os erros na medicação está a tecnologia de informação. E, muitas têm sido as estratégias utilizadas para minimizar potenciais falhas e ou erros no ciclo do medicamento, como o uso do código de barras nas embalagens dos medicamentos e a prescrição médica eletrônica ${ }^{19-22}$.

A prescrição médica eletrônica tem sido objeto de estudos, por ser aquela onde o médico (ou o transcritor), em vez de escrever manualmente a prescrição numa folha, utiliza um computador para digitá-la seguindo um modelo já estabelecido ${ }^{22-24}$.

Espera-se que a prescrição eletrônica possa reduzir a quantidade de erros, falhas potenciais, uma vez que elimina a dificuldade na leitura e no entendimento ocasionado pela letra ilegível do médico e possibilita que os erros de digitação sejam corrigidos no momento da elaboração da prescrição sem que, para isto, haja rasuras ou rabiscos que dificultam ainda mais o entendimento das informações ${ }^{(22-24)}$.

Observou-se no presente estudo, que a instituição/ hospital adota o sistema de prescrição eletrônica há quatro anos, mas os seus usuários prescritores continuam utilizando recursos de escritas manuais no impresso eletrônico gerado como prescrição.

Também não há uma agenda que permita a prescrição dentro do prazo de 24 horas, o que leva a atrasos de medicamentos, uma vez que o prescritor não realizou uma nova prescrição, dificultando o entendimento da enfermagem nos novos aprazamentos, que são diários enquanto o paciente está internado no hospital. Por parte da enfermagem, também existe uma falha nos aprazamentos, o que leva a não checagem da prescrição, mas que propicia atrasos, que podem levar a erros de medicação. Há também uma resistência no uso da prescrição eletrônica por parte dos prescritores, que trazem formulários impressos, pessoais, com a prescrição do paciente, que não é reconhecida como da instituição/ hospital, uma vez que o processo adotado é eletrônico.

Essas não conformidades talvez sejam consequência ao fato da instituição adotar um sistema de prescrição eletrônica que ainda permite que o médico faça registros manuais, principalmente naqueles casos de mudança da 
condição do paciente, suspensão de medicamentos ou inserção de novos medicamentos, e ou quando há médicos resistentes às ferramentas de tecnologia da informação. Também há o fato de que a enfermagem realiza o registro do medicamento administrado na própria folha impressa da prescrição e uma nova folha com outros medicamentos e ou suspensão dos atuais no mesmo dia poderia ser um fator a mais na ocorrência de erros, especialmente quase-falhas (near miss) relacionados ao atraso de medicamentos. Além do que, os auxiliares de enfermagem podem ter ainda a prática de seguir a uma cópia da prescrição emitida pela farmácia com os medicamentos dispensados e não seguir a que se encontra no prontuário do paciente, só a utilizando para registrar a administração no final do turno.

Um outro fator a ser considerado é que o registro da administração dos medicamentos é realizado na própria prescrição, e, quando o profissional pretende suspendê-lo e ou acrescentá-lo na folha, o faz manualmente para não ter que elaborar e ou imprimir uma nova prescrição, prática que pode levar a quase-falhas (near miss) /erros

Sabe-se que a implantação de Prontuário Eletrônico de Paciente não é uma tarefa fácil e de imediato uso adequado. Como tudo que gera mudanças, especialmente hábitos, o processo de implantação do uso de tecnologias não é um processo fácil e bem absorvido pelos seus usuários, que apresentam resistências o que leva ao insucesso ${ }^{21-27 .}$

Num estudo sobre o conhecimento das experiências relacionadas à implantação de prontuário eletrônico, observou-se como vantagens de um sistema informatizado de registros: o acesso rápido ao histórico do paciente; a facilidade na consulta de dados em atendimentos futuros; a redução no tempo de atendimento; a melhoria no controle e planejamento hospitalar e a melhoria na qualidade do atendimento. Por outro lado, como desvantagem citada por mais de $50 \%$ dos estudos selecionados foi a resistência dos profissionais da saúde quanto ao uso de novas tecnologias, o que se deve, normalmente, à falta de domínio de informática dos usuários. Fato este que sugere que o sucesso ou fracasso na implantação de um sistema de prontuários eletrônico está condicionado, diretamente, ao treinamento intenso e adequado da equipe e à sua participação nas diversas etapas que precedem a implantação do sistema e à familiaridade dos usuários com o sistema implantado ${ }^{27^{3}}$.

Não há como negar os avanços tecnológicos, em particular, o sistema computadorizado da prescrição da medicação como estratégia de redução da frequência de não conformidades/ erros no ciclo do medicamento/prescrição ${ }^{21}$ 27. Mas, deve-se estar atento para a ocorrência de alterações na prescrição eletrônica, que acabam sendo realizadas de forma manual, especialmente em situações de emergências onde o médico precisaria acessar o sistema para prescrever um medicamento de necessidade imediata, e isso levaria mais tempo em detrimento da prescrição manual. Também é importante lembrar que não existe um número de computadores disponíveis ideal nas instituições, não só pelo custo, mas também pelas dificuldades de espaços nas áreas de 
internação, além da dificuldade de acrescentar novas informações ao sistema quando necessárias, assim como a da inserção, manualmente, dos horários da administração do medicamento ${ }^{21-27}$.

$\mathrm{Na}$ formulação do FMEA realizada, a matriz de tolerância facilitou as atualizações e a visualização do status do risco relacionados às não conformidades identificadas de quase-falhas (near miss). A metodologia também mostrou-se de fácil utilização, permitindo identificar no processo analisado os pontos críticos e passíveis de monitoração por meio de indicadores relacionados às metas de segurança do paciente, assim como listar possíveis planos de ações de melhorias, com bases técnicas.

Através da matriz de tolerância do FMEA fica mais fácil implementar planos de ações específicos, para que a Instituição que adota um sistema de prescrição eletrônica ainda não informatizado, possa visualizar suas vulnerabilidades, $\mathrm{e}$ assim criar políticas e processos que melhorem os riscos existentes que levam a potenciais eventos/ erros no ciclo da medicação. Também permite que sejam criados gatilhos preventivos que impeçam possíveis não conformidades relacionadas ao ciclo do medicamento. Todas as medidas sugeridas a partir da matriz de tolerância do FMEA de atraso de medicamento, na prática, não são fáceis de serem modificados e sistematizados, pela complexidade, custos e resistências por serem consideradas pelas equipes multidisciplinares como ações "burocráticas". Mas poderão nortear às equipes naquilo que pode ser melhorado e implantado para evitar as não conformidades existentes que levam dia a dia à quase-falhas (near miss) de atraso de medicamentos.

Assim, observa-se também a necessidade de que sejam incluídas as oportunidades de melhorarias no sistema de prescrição eletrônica, quando este utilizado pela instituição de saúde/ hospital que contemplem: 1 - revisão das prescrições para evitar repetição; 2 - preenchimento de receituários no sistema; 3 - maior atenção do profissional ao prescrever; 4 - simplificação do programa; 5 - maior número de computadores, impressoras e manutenção destes; 6 - requisição informatizada de quimioterápicos e psicotrópicos, sem permitir a inclusão manual na prescrição eletrônica, entre outros.

É importante saber que a introdução da prescrição eletrônica ainda é algo novo na maioria das instituições de saúde/ hospitais do Brasil, e até mesmo em países com reconhecido desenvolvimento tecnológico, e por essa razão a sua implantação deve ter avaliações periódicas para que o sistema seja aperfeiçoado.

Há, entretanto, um futuro desenhado para o sistema de medicações nos hospitais onde o médico escreverá suas ordens em prescrições eletrônicas e receberá informações, via computador, sobre problemas como alergias e apoio à sua decisão na escolha do melhor tratamento. As prescrições serão enviadas eletronicamente pela farmácia e os farmacêuticos terão um papel de orientação clínica, a enfermagem terá máquinas para a dispensação de medicamentos e 
códigos de barra determinarão as doses administradas a quem, por quem e quando 25,26 .

Todos os aspectos aqui mencionados revelam a importância do estudo dessa temática e intervenções a partir de novas abordagens relacionadas, embora a tecnologia esteja sendo desenvolvida para auxiliar os profissionais a prevenirem o erro, é ainda o fator humano que está nas duas pontas do sistema entre o paciente e o profissional. Também é notada a multiplicidade de aspectos envolvidos na administração de medicamentos, quando não há mais lugar para ambientes punitivos diante de erros, uma vez que o que se busca é análise destes e suas prevenções.

A instituição do sistema de prescrição eletrônica e a parceria dos médicos na adequada utilização do mesmo representa um avanço na busca de estratégias para prevenção dos erros, e o envio dos dados diretamente à farmácia e a revisão dessa prescrição por um farmacêutico é outro aspecto a ser considerado.

Como aperfeiçoamento do sistema de prescrição eletrônica e com a educação por parte daqueles que prescrevem, as instituições deverão tornar as prescrições mais detalhadas, informativas, corretas e fáceis de serem compreendidas pelos profissionais que as manuseiam, já que este é um grande avanço nas estratégias para redução de eventos potenciais/ erros. Lembrando que a segurança do paciente e a melhoria da qualidade do serviço de saúde são metas que os profissionais têm e assim acredita-se que a prescrição eletrônica sendo bem utilizada pode contribuir no alcance dessas metas, evitando falhas potenciais (near miss) e ou erros para os pacientes.

\section{CONSIDERAÇÕES FINAIS}

É fundamental que existam políticas de melhorias que aumentem a segurança do ciclo dos medicamentos, especialmente, no atraso destes, que podem gerar quase-falhas (near miss) nas instituições que usam prontuário manual e principalmente eletrônico. De acordo com a matriz de tolerância das não conformidades identificadas relacionadas a atraso de medicamentos, o estudo mostrou que serão necessárias medidas que promovam: 1 -utilizar apenas prescrição eletrônica na instituição em 100\% dos pacientes; 2 - não permitir acréscimos/ registros manuais às prescrições eletrônicas; 3 - implantação de uma política institucional para os prazos de renovação da prescrição; 4 estabelecimento de definições de bloqueio do envio de medicamentos quando prescrições eletrônicas não estiverem conformes; 5 - realização de processo educacional continuado para as equipes de enfermagem, e outras, quanto a checagem de medicamentos e 6 - definição de uma política para avaliação das equipes multidisciplinares em casos reincidentes. Tais estratégias somente estarão coerentes dentro de um aspecto que privilegie a cultura de segurança na medicação e a melhoria da qualidade assistencial prestada à sua clientela, pacientes. 


\section{DECLARAÇÃO DE PARTICIPAÇÃO}

Sylvia Lemos Hinrichsen, Kaline Assis Carneiro Brayner, Silvio Luis Lira Paixão, Marcela Coelho Lemos e Ewerton Douglas da Silva contribuíram substancialmente para a concepção e planejamento do projeto, obtenção de dados ou análise e interpretação dos dados;

Sylvia Lemos Hinrichsen e Tatiana de Aguiar Santos Vilella contribuíram significativamente na elaboração do rascunho ou na revisão crítica do conteúdo.

Sylvia Lemos Hinrichsen e Tatiana de Aguiar Santos Vilella participaram da aprovação da versão final do manuscrito.

\section{REFERÊNCIAS}

1. Carvalho VT, Cassiani SHB, Chiericato C, Miasso Al. Erros mais comuns e fatores de risco na administração de medicamentos em unidades básicas de saúde. Rev Latino-am Enfermagem 1999; 7(5): 67-76.

2. Kawano DF, Pereira LRL, Ueta JM, Freitas O. Acidentes com medicamentos: como minimizá-los? Rev Bras Cienc Farm 2006; 42(4): 487-95.

3. Ministério da Saúde (BR). Programa do medicamento hospitalar. Brasília: Ministério da Saúde. Available from: <http://www.acss.min-saude.pt $>$ Acesso em 01/09/16

4. Bohomol E, Ramos LH. Erro de medicação: importância da notificação no gerenciamento da segurança do paciente. Rev Bras Enferm 2007; 60(1): 32-6.

5. Carvalho VT, Cassiani SHB. Erros na medicação e conseqüências para profissionais de enfermagem e clientes: um estudo exploratório. Rev Latino-am Enfermagem 2002; 10(4): 523-9.

6. Fontenele RE, Araujo TL. Análise do planejamento dos horários de administração de medicamentos em unidade de terapia intensiva cardiológica. Rev Enferm UERJ 2006; 14(3): 342-9.

7. Cassiani SHB, Teixiera TCA, Optiz SP, Linhares JC. O sistema de medicação nos hospitais e sua avaliação de um grupo de profissionais. Rev Esc Enferm USP 2005; 39(3): 280-7.

8. Oliveira RC, Camargo AEB, Cassiani SHB. Estratégias para prevenção de erros na medicação no setor de emergência. Rev Bras Enferm 2005; 58(4): 399-404.

RAS - Vol. 17, № 66 - Jan-Mar 2017 
9. JCl. Joint Commission International. Padrões de Acreditação da Joint Commission International para Hospitais. Manual. 5a Ed. 2014. 303p.

10. National Coordinating Council for Medication Error Reporting end Prevention. Taxanomy of medication errors 1998-1999. Available from: $<$ http://www.nccmerp.org >Acesso em 01/09/16

11. American Society of Health System Pharmacists. Suggested definitions and relationships among medication misadventures, medication errors, adverse drug events, and adverse drug reactions. Am J Hosp Pharm 1998; 55(2): 165-6.

12. Anacleto TA, Perini E, Rosa MB, César CC. Erros de medicação e sistemas de dispensação de medicamentos em farmácia hospitalar. Clinics 2005; 60(4): 325-32.

13. Cassiani SHB, Carvalho VT. Análise dos comportamentos dos profissionais de enfermagem frente aos erros na administração de medicamentos. Acta Paul Enferm 2002; 15(2): 45-54.

14. Coimbra JAH, Cassiani SHB. Responsabilidade da enfermagem na administração de medicamentos: algumas reflexões para uma prática segura com qualidade de assistência. Rev Latino-am Enfermagem 2001; 9(2): 56-60.

15. Hinrichsen; SL. Qualidade \& Segurança do Paciente. Gestão de Riscos. Rio de Janeiro: Medbook. 2012. 335p.

16. Wacheter; RM. Compreendendo a Segurança do Paciente. Porto Alegre: Artmed. 2010. 320p.

17. Hinrichsen SL, Possas L, Oliveira CLF, Ramos DM, Vilella TAS. Análise de Modos e Efeitos de Falhas (FMEA) e metas internacionais de segurança do paciente: estudo-piloto. RAS 2012; 14 (57): 151-60.

18. Borges MR, Perini E, Anacleto TA, Neiva HM, Bogutchill T. Erros na prescrição hospitalar de medicamentos potencialmente perigosos. Rev Saúde Pública 2009; 43(3): 490-8.

19. Aspden P, Wolcott J, Bootman JL, Cronenwett LR. Committee on Identifying and Preventing Medication Errors. Preventing medication errors. Quality Chasm Series (Hardcover). Washington: National Academies Press 2007. 480p.

20. Barber N, Rawlins M, Dean Franklin B. Reducing prescribing error: competence, control, and culture. Qual Saf Health Care. 2003; 12 (1): 29-32.

21. Ghandi TK, Seger DL, Bates DW. Identifying drug safety issues: from research to practice. Health Care Int J Qual 2000; 12(1): 69-76. 
22. Shane R. Computerized physician order entry: challenges and opportunities. Am J Health Syst Pharm 2002; 59: 286-88.

23. Payne; TH. Computer decision support systems. Chest 2000; 118(2): 47S$52 \mathrm{~S}$.

24. Wynn; P. Get ready! Electronic prescriptions are coming. Managed Care Magazine 1996; 5: 44-8.

25. Bates; DW. Improving medication safety across institutions. J Qual Improv 2000; 26(6): 319-20.

26. Cassiani SHDB, Freire CC, Gimenes FRE. A prescrição médica eletrônica em um hospital universitário: falhas de redação e opiniões de usuários. Rev Esc Enferm USP 2003; 37(4): 51-60.

27. Canêo PK, Rondina JM. Prontuário Eletrônico do Paciente: conhecendo as experiências de sua implantação. J Health Inform 2014 6(2): 67-71. 